\title{
Full 3D Dispersion Curve Solutions for Guided Waves in Generally Anisotropic Media ${ }^{0}$
}

\author{
F. Hernando Quintanilla ${ }^{a}$, M. J. S. Lowe ${ }^{a}$ and R. V. Craster ${ }^{b}$ \\ ${ }^{a}$ Department of Mechanical Engineering, ${ }^{b}$ Department of Mathematics, Imperial College, \\ London $S W 7$ 2AZ, UK
}

\begin{abstract}
Dispersion curves of guided waves provide valuable information about the physical and elastic properties of waves propagating within a given waveguide structure. Algorithms to accurately compute these curves are an essential tool for engineers working in non-destructive evaluation and for scientists studying wave phenomena. Dispersion curves are typically computed for low or zero attenuation and presented in two or three dimensional plots. The former do not always provide a clear and complete picture of the dispersion loci and the latter are very difficult to obtain when high values of attenuation are involved and arbitrary anisotropy is considered in single or multi-layered systems. As a consequence, drawing correct and reliable conclusions is a challenging task in the modern applications that often utilise multi-layered anisotropic viscoelastic materials. These challenges are overcome here by using a spectral collocation method (SCM) to robustly find dispersion curves in the most complicated cases of high attenuation and arbitrary anisotropy. Solutions are then plotted in three-dimensional frequency-complex wavenumber space, thus gaining much deeper insight into the nature of these problems. The cases studied range from classical examples, which validate this approach, to new ones involving materials up to the most general triclinic class for both flat and cylindrical geometry in multi-layered systems. The apparent crossing of modes within the same symmetry family in viscoelastic media is also explained and clarified by the results. Finally, the consequences of the centre of symmetry, present in every crystal class, on the solutions are discussed.
\end{abstract}

\footnotetext{
${ }^{0}$ Accepted for publication in the Journal of Sound and Vibration,
} 


\section{Introduction}

In the study, and engineering application, of elastic guided waves the dispersion curves of waves propagating within a given structure are a vital tool for inspection purposes and for obtaining information about the properties of the material itself. Dispersion curves for propagating and non-propagating modes are often computed for zero, or low, attenuation values and presented as two or three dimensional plots. See for instance [1], [2], [3] or more recently [4], [5] and [6]. However, cases where high values of attenuation are present and arbitrary anisotropy in single or multi-layered structures is considered have been hardly studied due to the great difficulties encountered when computing their dispersion curves. For instance, dispersion curves for viscoelastic monoclinic plates have been found and, for low values of the attenuation, presented in two dimensional plots [7], but in general, a better understanding of the nature of the modes, and a clearer visualization of the solutions, is achieved when dispersion loci are reliably found for low as well as high values of attenuation and therefore dispersion curves are required in three dimensions.

In the literature, modes have been categorised in a number of ways and here the classification of Auld [8] is used: propagating modes have real wavenumber, for this reason they are often the most useful for engineering application in NDT since they propagate and transport energy within the structure without attenuation. The second type of solution is non-propagating modes with complex or purely imaginary wavenumber. Note that these modes with complex or purely imaginary wavenumbers are also present in perfectly elastic materials without any energy leakage, those solutions represent local modes that would exist at discontinuities. In addition, non-propagating modes are of interest in certain applications such as the solution of scattering problems using modal summations where a knowledge of the full set of possible modes is essential, [9]. A detailed discussion about the physical properties and energy transportation of non-propagating modes in elastic (lossless) media is in the second volume of Auld [8]. For viscoelastic materials, only propagating modes with complex wavenumber exist lest unphysical results are obtained as pointed out in [6]. Propagating modes in viscoelastic media can in turn be split into: lowly attenuated modes, with almost real wavenumbers and

DOI:10.1016/j.jsv.2015.10.017, November 2015. 
therefore very useful for inspection purposes in NDT; and highly attenuated modes, with dominant imaginary part for the wavenumbers. The reader must be aware that there does not seem to be a consensus about this nomenclature, see for example [8], [10] and [11]. In this paper we adopt the convention by Auld [8] and we will try to make it clear what kind of modes we are talking about in each case by specifying the nature of the wavenumber. Collectively, these types of modes constitute the complete spectrum for a given problem and their study and visualization is the object of our current investigation.

Several methods have been developed, and successfully deployed, to compute two-dimensional, and less often three-dimensional, dispersion curves for guided waves in flat and cylindrical geometry: finite element (FE), semianalytical finite element (SAFE) simulations, root-finding routines based on the partial wave approach (Partial Wave Root Finding, PWRF) and lately, spectral methods have become more popular as a powerful alternative, see [4], [12] or [13]. More recently, one of these variants known as Spectral Collocation Method (SCM) has been successfully used to model guided waves in elastic and viscoelastic generally anisotropic media, [14] and [15] respectively.

The main contribution and novelty of the present paper is the deployment of a SCM to compute the full three-dimensional spectrum, including highly attenuated and non-propagating purely imaginary modes, for guided wave problems in three cases (Figures 4.a, 5.a, 7 and 9.) which, to the best of the authors' knowledge, have not been studied before and comprise up to the most general type of anisotropy, namely triclinic crystals. Several other wellknown cases have also been presented for validation purposes. Regardless of the case under study, this investigation also emphasizes that with the SCM no additional coding effort is needed to obtain the complete three-dimensional spectrum which contrasts with conventional approaches for which the algorithms become significantly more inefficient when searching in a threedimensional space and which may require substantial modification to obtain robust solutions. The SCM used here was first presented in a previous work by Hernando et. al [15], though there, attention was restricted solely to lowly attenuated modes. The present work goes one step further and, by carefully sorting the eigenvalues provided by the SCM, completes the picture giving the remaining branches of the dispersion loci for the most general anisotropic triclinic class. It is notable that with technological advances there is a need for this full anisotropic generality and for the robust generation of the corre- 
sponding three-dimensional dispersion curves presented in this paper for the first time.

Additional contributions of this paper, arising from the undertaken investigations, are the clarifications concerning the apparent crossing of modes within the same symmetry family for viscoelastic media (Section III) and the discussion of the implications of the centre of symmetry of all crystal classes for the solution (Section V).

The paper is organized as follows. In section 2 a very brief description of the SCM, and its implementation is given, this is kept to a minimum to avoid repetition and the reader is referred to [12], [14], [15] and references therein for all the details about the SCM scheme and an extensive discussion of its principal features when used to solve acoustic guided wave problems. Some modifications are required here and these are outlined. In section 3, classical examples in flat geometry both for elastic and viscoelastic media are presented as a further validation of the SCM approach. The crossing of modes, which is an important detail in practice, is discussed and elucidated with the aid of a new three-dimensional plot for the dispersion curves of a monoclinic material. The full solution for a new multilayer flat case is presented at the end of section 3. Section 4 treats cylindrical geometry, firstly a classical example is briefly presented for validation and the section closes with a new illustrative multilayer cylindrical system. Section 5 is devoted to the discussion of the results and conclusions.

\section{SCM methodology outline}

A comprehensive description of the SCM is in [12], [14] and, with particular relevance to the present case, in [15]. The main idea behind the SCM is to convert the partial differential equations of the acoustic field into a generalized matrix eigenvalue problem whose eigenvalues are computed thus yielding the full spectrum. We highlight some minor modifications regarding the post processing of results which must be done in order to visualize the full three-dimensional solution.

The governing equations of motion for a linear elastic anisotropic homogeneous medium are:

$$
\nabla_{i K} c_{K L} \nabla_{L j}^{s y m} u_{j}=-\rho \omega^{2} u_{i}
$$


with the summation convention over the indices and $c_{K L}$ is the medium's stiffness matrix in reduced index notation, [8], $u_{j}$ are the components of the displacement vector field that for the application here are

$$
u_{j}=U_{j}(y) e^{i(k z-\omega t)} ; \quad j=x, y, z
$$

The differential operators in (1) are of first order in the coordinate derivatives and their explicit expressions are in [8]. The expression of the stress tensor field in terms of the strain tensor field, $S_{k l}$, for a perfectly elastic material reads:

$$
T_{i j}=c_{i j k l} S_{k l}
$$

here $c_{i j k l}$ is the fourth-rank stiffness tensor related to $c_{K L}$ as described in [8]. When material damping is included, that is, when modelling viscoelastic media, the viscoelastic stress tensor field takes the following more complex form assuming the Kelvin-Voigt frequency dependent model (see [15] for details) to account for material damping:

$$
\tilde{c}_{p j k l}^{K V}=c_{p j k l}-i \frac{\omega}{\hat{\omega}} \eta_{p j k l}
$$

Where $c_{p j k l}$ and $\eta_{p j k l}$ have the same units and the prefactor $\omega / \hat{\omega}$ is nondimensional. The $\eta_{p j k l}$ tensor accounts for losses within the material. A more detailed description of the derivation of this expression is in Auld [8]. For structures in a vacuum, traction-free boundary conditions hold. These require the vanishing of the following three components of the stress tensor field. Taking the faces of the plate to be located at $y= \pm h / 2$ the boundary conditions (BCs) are given by:

$$
\left.T_{y y}\right|_{y= \pm h / 2}=\left.T_{y x}\right|_{y= \pm h / 2}=\left.T_{y z}\right|_{y= \pm h / 2}=0 .
$$

Turning now to the implementation of the SCM, the standard procedure consists of discretizing the physical domain and replacing the derivatives in Eq. (1) by the discrete Differentiation Matrices (DMs). For a single layer in vacuum, since we have a bounded interval, an appropriate, spectrally accurate, choice is to use Chebyshev DMs, based on a non-uniform Chebyshev grid of $N$ points, these are $N \times N$ matrices; the generation of DMs is covered 
in [16], [17]. The $m$-th derivative with respect to $y$ is approximated by the corresponding $m$-th order Chebyshev $N \times N$ DM:

$$
\frac{\partial^{(m)}}{\partial y^{(m)}} \Longrightarrow \mathbf{D}^{(m)}:=\left[D M_{C h e b}\right]_{N \times N}^{(m)}
$$

The substitution is made in both the equations of motion Eq. (1) and boundary conditions Eq. (5) yielding the matrix analogue that is concisely written as:

$$
\mathfrak{L}(k) \mathbf{U}=\omega^{2} \mathfrak{M} \mathbf{U}
$$

where $\mathbf{U}$ is the vector of vectors: $\mathbf{U}=\left[\mathbf{U}_{x}, \mathbf{U}_{y}, \mathbf{U}_{z}\right]^{T}$, these vectors $\mathbf{U}_{i}$ are the components of the displacement vector field. The matrix $\mathfrak{L}(k)$ contains the differential operators of the PDEs and the matrix $\mathfrak{M}$ is the identity multiplied by $-\rho$. The boundary conditions (BC) in Eq. (5) are taken into account by appropriately substituting them in the corresponding rows of Eq. (7) after having recast them in matrix form, in the same fashion as was done for the equations of motion, this procedure is described in detail in [14].

The $\mathfrak{L}(k)$ matrix contains terms proportional to $k^{0}, k^{1}$ and $k^{2}$. Rearranging the terms in the matrix equation Eq. (7) and decomposing the $\mathfrak{L}(k)$ matrix so that the $k$ dependence of the different terms becomes more apparent leads to the following expression of Eq. (7):

$$
\left(\mathbf{Q}_{2} k^{2}+\mathbf{Q}_{1} k+\mathbf{Q}_{0}\left(\omega^{2}\right)\right) \mathbf{U}=0
$$

Now it should be clear that, once we fix the value of $\omega$, this does not have the structure of a general eigenvalue problem in $k$. In order to achieve this structure (see Eq. (7)), a mathematical technique known as the Linear Companion Matrix Method is used, see [18], [19] or [15]. Once we recast this again in the form of Eq. (7) which is a typical generalized eigenvalue problem, it is easily solved using an eigensolver routine that yields the complex eigenvalues $k$; the cases presented here were solved with the routine eig of MATLAB (version R2012b).

The SCM is known to produce some spurious eigenvalues as pointed out in [20] and [12] for instance. The methodologies to deal with them have been extensively discussed there and a similar discussion will not be pursued here. These general procedures apply equally in the present case but in viscoelastic materials the full solution comprises all possible modes described 
in the introduction: purely real, purely imaginary (only present in the perfectly elastic case) and complex. Therefore no physical eigenvalues will be discarded as would be done if one was only interested in propagating modes with low attenuation. The same filter as used in [15] to retain only propagating modes is deployed to classify the different solutions. The filter uses the ratio between the real and imaginary parts of $k=\alpha+i \beta$ defined as:

$$
R \equiv \frac{\beta}{\alpha}
$$

Once this filter is introduced the classification of the modes is rather straightforward. For the elastic case, they are split into the aforementioned categories according to the nature of the wavenumber: propagating modes with real wavenumber and non-propagating modes with complex wavenumber and non-propagating modes with imaginary wavenumber. For the viscoelastic case, only propagating modes exist and these are split into two categories as indicated earlier, lowly and highly attenuated modes. Since now all the modes have complex wavenumber, once a value of $R$ is fixed and for a given range of wavenumbers, the modes are split into those with a higher value of the wavenumber's imaginary part, propagating strongly attenuated modes, and those with a lower value that are propagating but less attenuated modes. In some regions of the spectrum of viscoelastic materials the wavenumber is almost purely imaginary and in figures this will be indicated, when necessary, with a different colour for clarity.

\section{Solutions for Systems in Flat Geometry}

The first case to consider is for the symmetric Lamb modes of a flat isotropic perfectly elastic free plate, [1], later reproduced in numerous textbooks on the subject such as [8] and [10]. Fig.1 shows the dispersion curves obtained by the SCM. Its excellent agreement with those shown in the original paper by Mindlin was checked by the authors and no difference was found between the two sets. Therefore, Mindlin's results are not reproduced here but can be found in his paper [1] or in any of the aforementioned more recent references. This serves as a further validation of the SCM approach. A complete description of the validation of the SCM for the study of guided waves in anisotropic media, elastic and viscoelastic, is in [14] and, of particular importance to the present case, in [15]. 


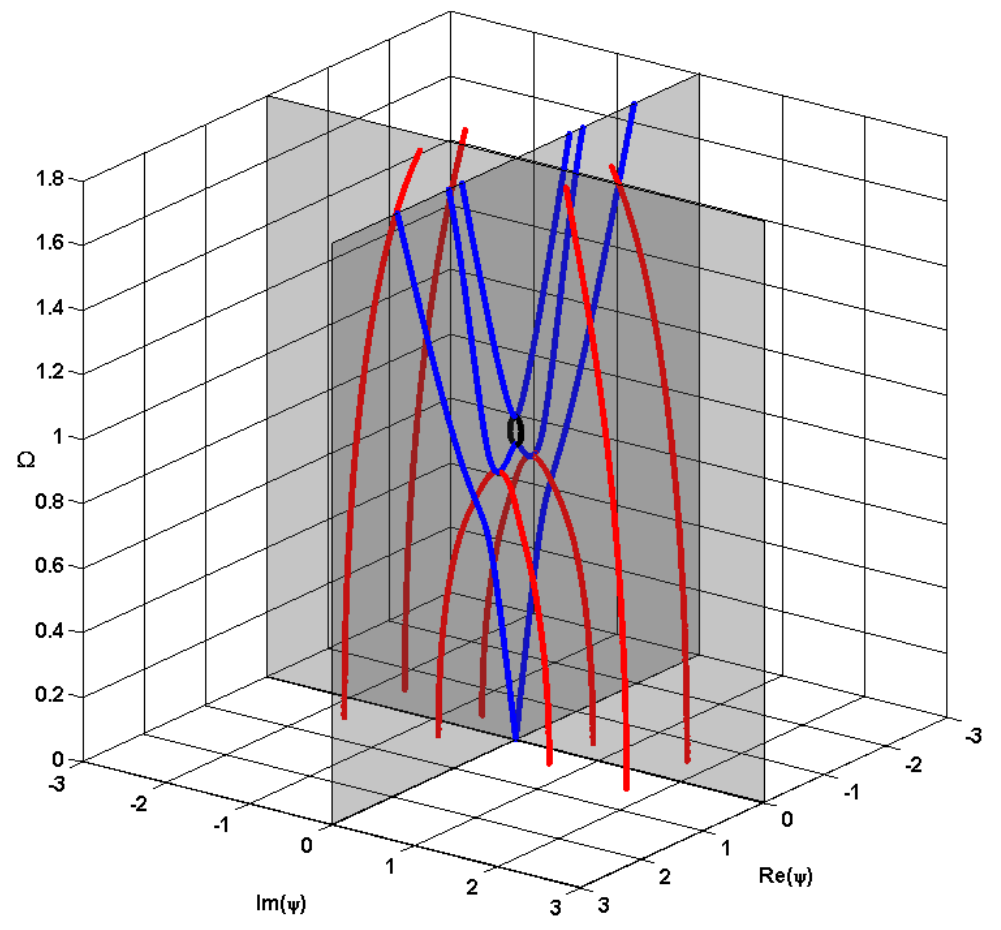

Figure 1: First three symmetric Lamb modes in a free elastic steel plate computed with the SCM. Propagating modes (lowly attenuated modes) in blue. Non-propagating highly attenuated modes in red and black (purely imaginary). The non-dimensional axes are $\Omega$ $=h \omega / \pi V_{66}$ and $\Psi=h k / \pi$. 
Throughout this section the conventional non-dimensional axis convention is adopted for the three-dimensional plots. The non-dimensional frequency is defined as follows:

$$
\Omega=\frac{h \omega}{\pi V_{66}}
$$

where $\omega$ is the angular frequency, $h$ is the half thickness of the single plate or the thickness of the multilayer system and $V_{66}=\sqrt{C_{66} / \rho} . \quad C_{66}$ is the corresponding entry in the stiffness matrix and $\rho$ is the density of the medium. $V_{66}$ reduces to the customary $c_{2}$ for isotropic media. The non-dimensional wavenumber is similarly defined as:

$$
\Psi=\frac{h k}{\pi}
$$

where $k$ is the wavenumber of the wave.

Next we turn our attention to a viscoelastic isotropic free steel plate, the symmetric and antisymmetric Lamb modes are shown separately in different projections. The wave propagates within the plane of the plate. In Fig.2.a and Fig. 2.b the full solutions for symmetric and antisymmetric modes are shown respectively with the same colour scheme as in Fig.1 but no regions of the spectrum have been coloured in black (strictly speaking, only the points at which the loci intersect the plane $\operatorname{Re}(\Psi)=0$ would be in black) to highlight that now all modes are propagating with higher or lower values of attenuation, and none with zero attenuation; in absorbing media all modes are of the propagating kind lest we obtain unphysical results, this has been discussed in detail in section 3 of [6]. In Fig.3.a and Fig.3.b projections of the symmetric and antisymmetric Lamb modes onto the non-dimensional Frequency-Real Wavenumber plane are presented for detail, and for comparison with those given in [6]. The set of dispersion curves shown in Fig.3. computed with the SCM have been found to present very good agreement with those in [6] which are not reproduced here for being identical to those in Fig.3, the original figures can be found in the aforementioned reference. Note that all axes in the present figures are rescaled by a factor $1 / 2$ due to the definition of the non-dimensional constants Eq.(10) and Eq.(11) where the half thickness $h$ has been used instead of the total thickness of the plate $2 h$ as it was done in [6]. The choice of colouring makes it clear when a branch of a mode runs in front of the branch of a different mode in three-dimensional space. The physical properties of this, and the previous example, are in the appendix. 
(a)

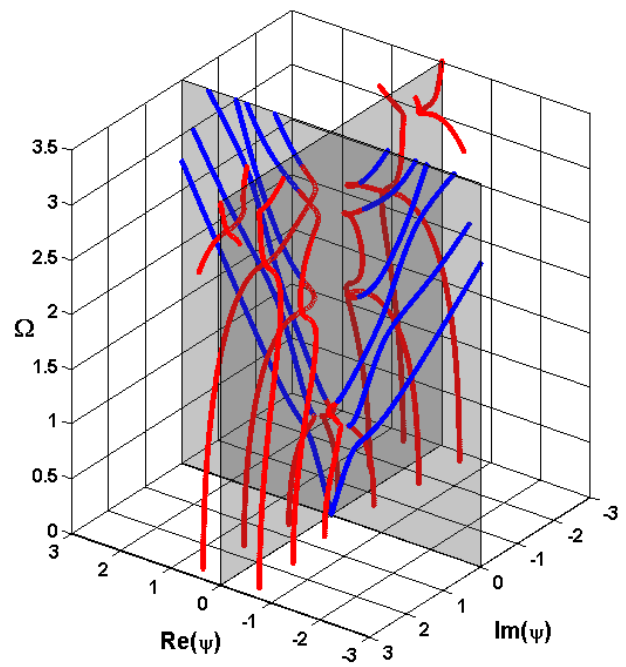

(b)

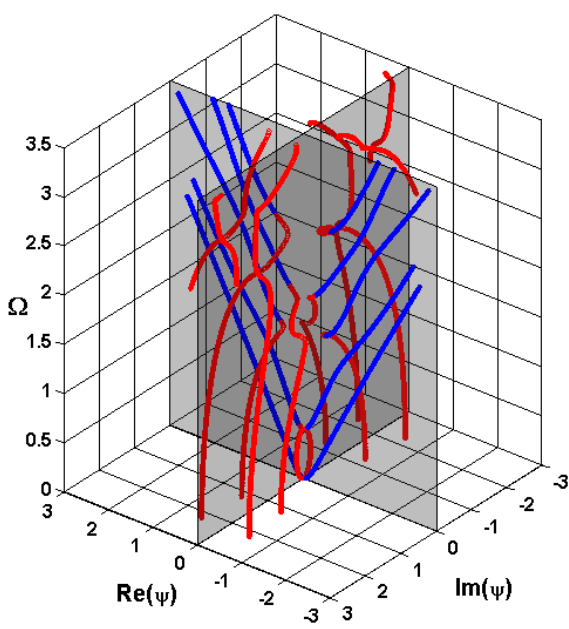

Figure 2: Symmetric (a) and Antisymmetric (b) Lamb modes in a free hysteretic-type viscoelastic steel plate computed with the SCM. Propagating modes in blue (lowly attenuated) and red (highly attenuated). Non-dimensional axes: $\Omega=h \omega / \pi V_{66}$ and $\Psi=h k$ $/ \pi$.

It is important to note already that the apparent crossing of modes within the same family, something forbidden in the elastic case, does not actually take place in the viscoelastic case either. This is explained by noticing that for the viscoelastic case one still has two independent dispersion relations for Lamb modes whose entries are now complex, thus yielding generally complex roots: See also Rose [11] or Graff [10] for a more detailed discussion about this. The splitting of modes into families of symmetric and antisymmetric Lamb modes is related to the geometrical symmetries of the crystal and axes configuration for the crystal chosen in each case, not to the dynamical properties of the material itself such as damping. Therefore, regardless of the material being elastic or viscoelastic, if the crystal configuration is such that modes can be classified according to their symmetry with respect to the middle plane of the plate, those two families will remain independent from each other for any kind of media and no crossing between them will occur. This is seen by looking at the full $3 D$ picture of the dispersion curves and noting 
(a)

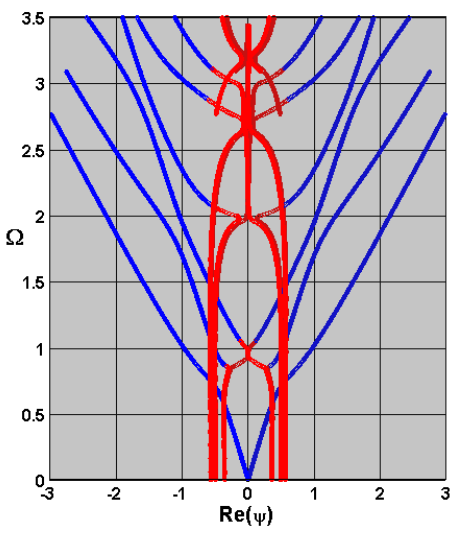

(b)

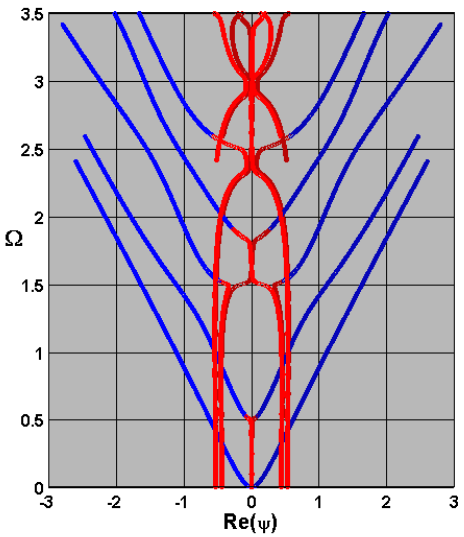

Figure 3: 2D projection of the symmetric (a) and antisymmetric (b) Lamb modes in a free hysteretic-type viscoelastic steel plate computed with the SCM shown in figure 2 . Propagating modes in blue (lowly attenuated) and red (highly attenuated).

that what seems to be a crossing in the $2 D$ projection is nothing more than one line passing in front of the other in the complete and clearer $3 D$ picture. This is a recurrent point of discussion in the literature, to cite only a few, see for instance [21], [22] and [23], it has also been addressed in [6] and in [5] for elastic plates with fluid loading, further references to other related papers can be found therein. The next example will further verify this assertion in the case of a more complex material.

Now a free viscoelastic plate of monoclinic material is investigated. The crystal configuration is as follows: the $\{Y\}$ axis of the crystal is normal to the plane of the plate and propagation takes place along the $\{Z\}$ axis, further details about the physical properties of the material are in the appendix. Fig. 4.a and Fig. 5.a present the full three-dimensional solution for symmetric and antisymmetric coupled modes respectively in a monoclinic plate which, to the best of the author's knowledge, has not been presented before. Its corresponding two-dimensional plots for propagating modes and attenuation are shown in Fig.4.b and Fig.4.c for symmetric modes and in Fig.5.b and Fig.5.c for antisymmetric modes respectively. It is shown in Fig. 
4.b that two of the symmetric modes, labelled as B and C, cross at roughly $0.50 \mathrm{MHz}$, a similar result was obtained and discussed in figure 5 of [7] for a different monoclinic plate. Note that the modes labelled A and B do not cross. Once more, it is worth emphasizing that no real crossing occurs in this case for the same reasons mentioned in the previous paragraph. For instance, Li and Thompson [24] derived the two generalized Lamb dispersion relations for symmetric and antisymmetric modes in a monoclinic plate with propagation within the plane of symmetry of the crystal, these were also derived in $[25]$.

These two independent dispersion relations derived in [24] hold also for the case of viscoelastic materials and therefore, having only one dispersion relation which cannot be further factorized into a simpler product of factors for each independent family, no crossings can occur amongst modes given by the corresponding non-reducible dispersion relation for the independent family under study. The fact that these dispersion relations cannot be further factorized has a physical interpretation as explained by Solie and Auld [26]: each dispersion relation is the determinant of the coefficient matrix of a set of equations whose unknowns are the amplitudes of the partial waves which give rise to the family of modes considered and the order of this non-reducible determinant is equal to the minimum number of partial waves needed for satisfying the boundary conditions. The six partial waves will be coupled by the boundary conditions and anisotropy of the crystal in various ways which depend on the problem under study. Note that the partial waves are mentioned here because they are a known valid means of representing the guided wave fields, and are thus a useful tool to aid the explanation, but it must be emphasised that partial waves are not part of the alternative SCM approach presented in this work. In the present case, three partial waves are coupled (coupling symmetric SH and Lamb modes) to give rise to the coupled symmetric modes, analogously for antisymmetric modes. Similar considerations and explicit formulae for the monoclinic case can be found also in [27].

The assertion that no crossings occur and the solution given by each of these non-reducible dispersion relations is unique can be explained as follows: Firstly, the non-reducibility of an $N \times N$ dispersion determinant implies that one needs at least as many partial waves as the order of the determinant to satisfy the boundary conditions, that is, $N$. Secondly, in order to have nontrivial solutions one requires the $N \times N$ determinant to vanish, this means 
(a)

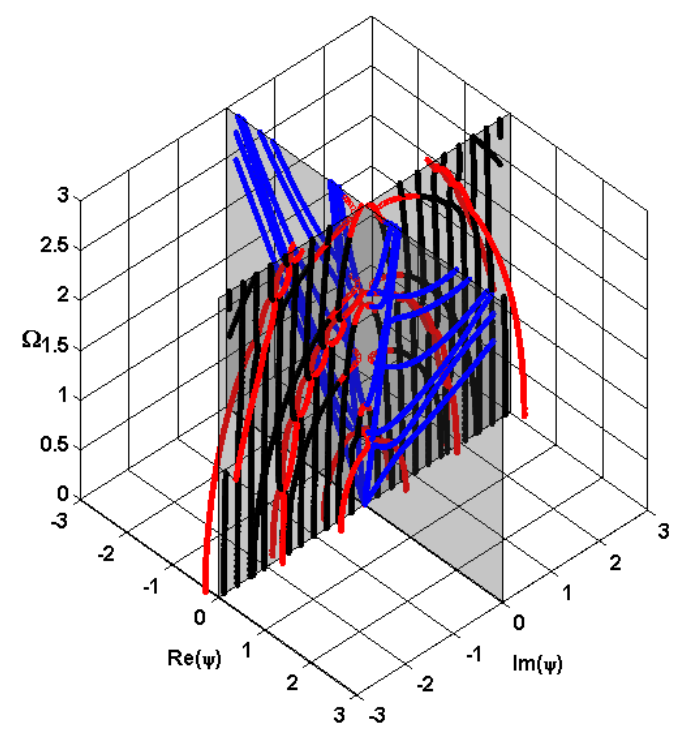

(b)

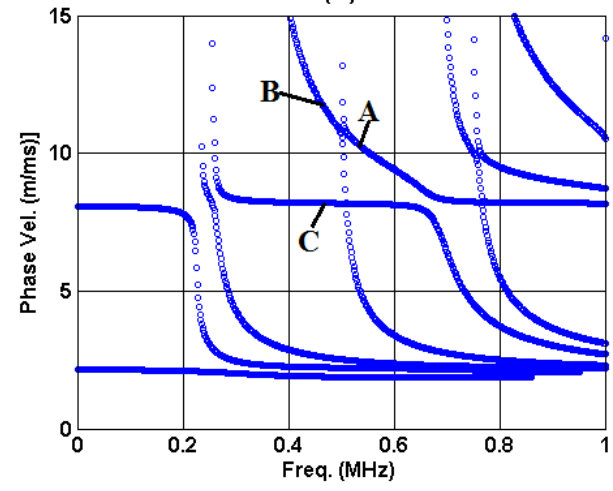

(c)

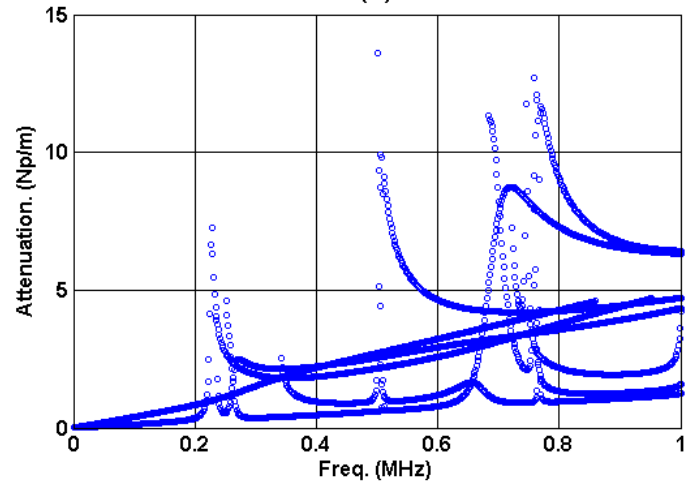

Figure 4: Top: Symmetric generalized Lamb modes in a free hysteretic-type viscoelastic monoclinic plate computed with the SCM (a). Propagation is along the $\{\mathrm{Z}\}$ axis, and the $\{\mathrm{Y}\}$ axis is perpendicular to the plane of the plate. Propagating Modes: low attenuated modes in blue, highly attenuated modes in red and almost imaginary wavenumber modes in black. Plot non-dimensional axes: $\Omega=h \omega / \pi V_{66}$ and $\Psi=h k / \pi$. Bottom: 2D dispersion curves (b) and attenuation (c) of the symmetric generalized Lamb modes in the viscoelastic monoclinic plate. Note the apparent crossing of modes B and C in (b) at roughly $0.5 \mathrm{MHz}$. 


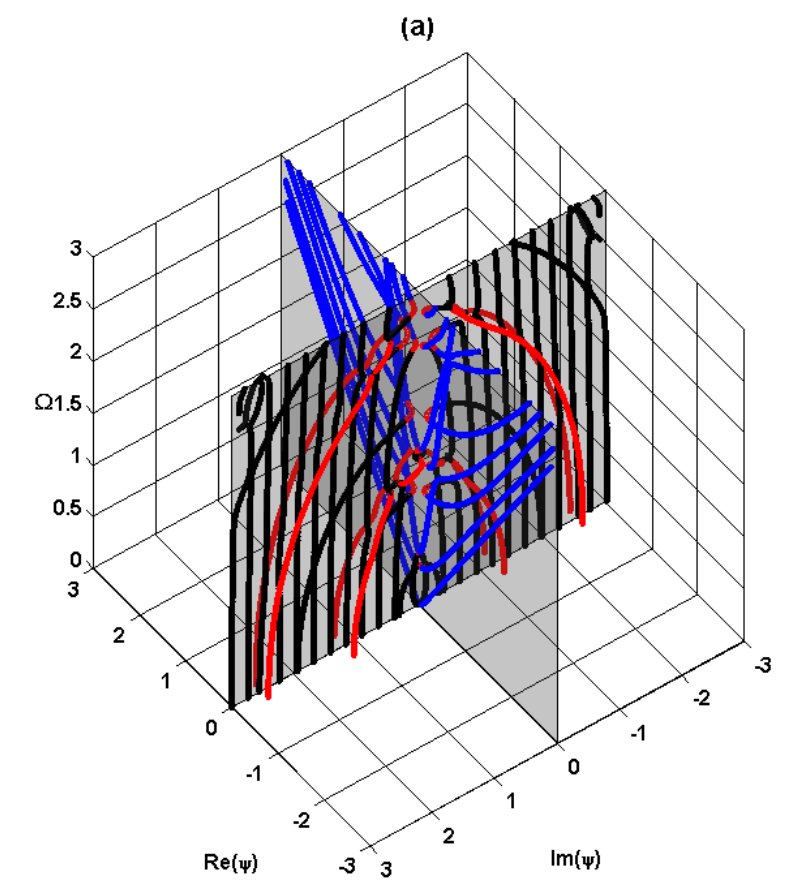

(b)

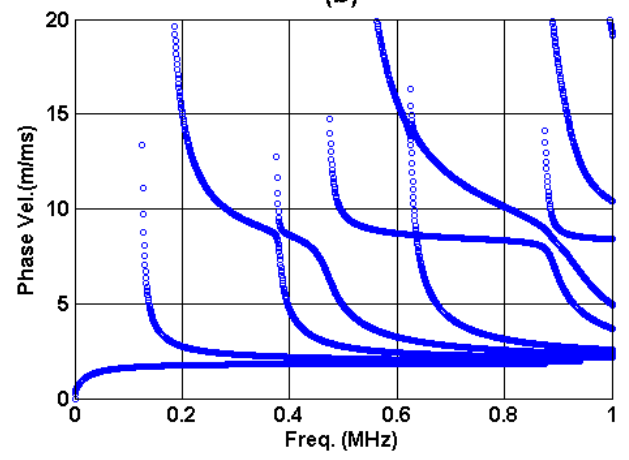

(c)

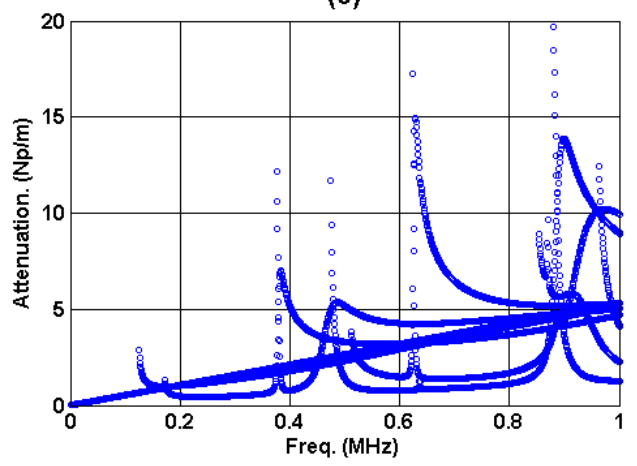

Figure 5: Top: Antiymmetric generalized Lamb modes in a free hysteretic-type viscoelastic monoclinic plate computed with the SCM (a). Propagation is along the $\{\mathrm{Z}\}$ axis, and the $\{Y\}$ axis is perpendicular to the plane of the plate. Propagating Modes: low attenuated modes in blue, highly attenuated modes in red and almost imaginary wavenumber modes in black. Plot non-dimensional axes: $\Omega=h \omega / \pi V_{66}$ and $\Psi=h k / \pi$. Bottom:2D dispersion curves (b) and attenuation (c) of the antisymmetric generalized Lamb modes in the viscoelastic monoclinic plate. 
the rank of the determinant is no bigger than $(N-1)$.

It can be seen that the rank is in fact $(N-1)$ thus yielding a unique nontrivial solution by the following consideration: if this was not so, and let us assume for definiteness that it was $(N-2)$, one could find non-trivial solutions expressing $(N-2)$ partial wave amplitudes in terms of the two remaining ones. In particular, one could set one of these latter amplitudes to zero while still having a non-trivial solution constructed from $(N-1)$ partial waves. This is in stark contradiction with the need for at least $N$ partial waves implied by the non-reducibility of the $N \times N$ dispersion determinant.

Interestingly, this can be confirmed by the SCM which uses an algebraic approach. It has been observed that for each independent family of modes the multiplicity of the eigenvalues, roots of the dispersion relation, is one. This means that the eigenspace spanned by the corresponding eigenvector (mode) is one-dimensional, which is tantamount to saying that only one dispersion curve runs through that point. Moreover, when a code for the most general case of triclinic is used to study the dispersion curves in an isotropic plate, the four independent families of modes (Symmetric/Antisymmetric Lamb and SH) are obtained and, up to the accuracy allowed by computer precision, it can be seen that eigenvalues with multiplicity two are obtained for the crossing points. Their two eigenvectors are orthogonal which means there are two different modes for that given eigenvalue: for instance an $\mathrm{SH}$ and a Lamb mode. In Fig.6, a detail of the problematic region in Fig.4.b around $0.50 \mathrm{MHz}$ is presented in three-dimensional space and it is seen very clearly that these two modes do not cross.

Finally for flat geometry we consider a bilayered system composed of a viscoelastic triclinic plate and an elastic orthorhombic plate. Figure 7 shows its three-dimensional complete spectrum which, to the best of the author's knowledge, has not yet been published for this or similar systems involving crystals of triclinic symmetry. The axis configuration is the same as in the previous example: the $\{Y\}$ axis of the crystal is normal to the plane of the plates and the propagation takes place along the $\{Z\}$ axis. More details about the physical properties of the plates are given in the appendix. This highlights the usefulness of the SCM approach for calculating the full spectrum of complicated cases of single or even multi-layered systems with different viscoelastic materials or combinations of elastic/viscoelastic mate- 


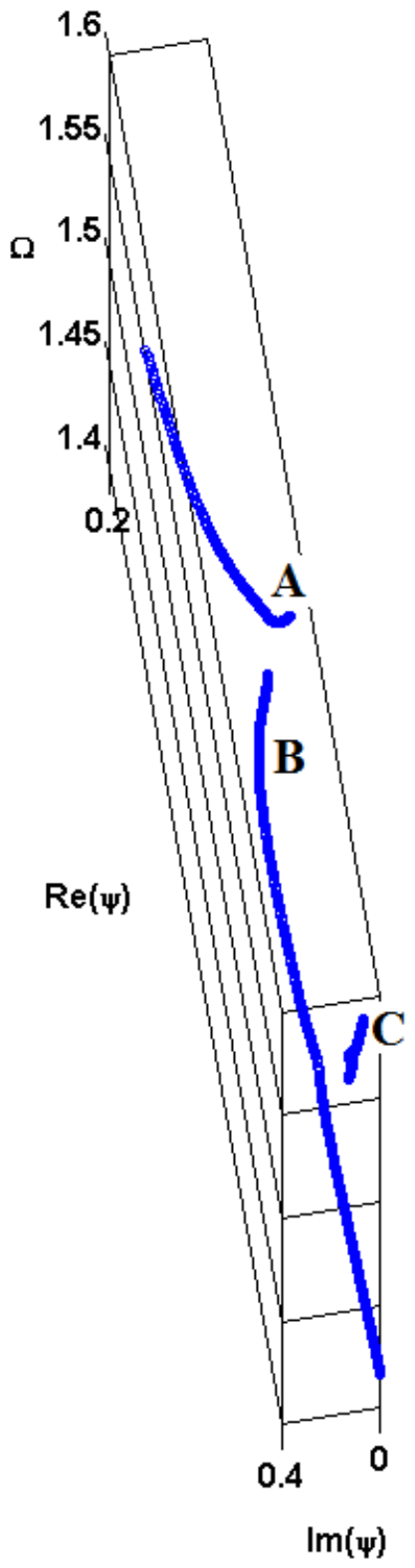

Figure 6: 3D detail of the region where the apparent crossing of symmetric generalized Lamb modes B and C in figure 4 (b) appears. When shown in three-dimensional space under the appropriate angle it is seen that actually no crossing between modes B and C occurs. 
rials. Similar solutions for much simpler systems are sometimes incomplete when they are computed by conventional root-finding routines for instance, which highlights the importance of the methodology and results shown in the present work. As a consequence of this, some of the modes might also appear to merge with each other. These difficulties are easily overcome by using a SCM that provides, without any extra coding effort, the full threedimensional solution; a detailed discussion of further advantages of the SCM for guided wave problems is given in [12], [14] or [15]. The choice of materials, in particular the triclinic layer, has been done in order to provide the solution for as general a case as possible, thus a triclinic layer has been included. Note that in the work by Hernando et. al [15] only the conventional two dimensional dispersion curves for lowly attenuated modes was presented though not a complete solution as the one given here.

\section{Solutions for Systems in Cylindrical Geometry}

Only two examples are shown in this section since the procedure to obtain the full solution in cylindrical geometry follows exactly the same lines as that for flat plates. In addition, a detailed study of these systems for nonattenuating and low-attenuating modes has been already carried out in [15]. Therefore, a classical example from the literature and a sufficiently general new case suffices to illustrate the capabilities of the SCM when obtaining full solutions in cylindrical geometry.

Throughout this section for cylindrical geometry, the conventional non-dimensional axes convention is adopted for the three-dimensional plots. The non-dimensional frequency is defined as follows:

$$
\Omega=\frac{a \omega}{V_{66}}
$$

where $\omega$ is the angular frequency, $a$ is the thickness of the cylindrical shell

system or the radius of the rod and $V_{66}=\sqrt{C_{66} / \rho} . C_{66}$ is the corresponding entry in the stiffness matrix and $\rho$ is the density of the medium. $V_{66}$ reduces to the customary $c_{2}$ for isotropic media. The non-dimensional wavenumber is similarly defined as:

$$
\Psi=a k
$$

where $k$ is the wavenumber of the wave. 


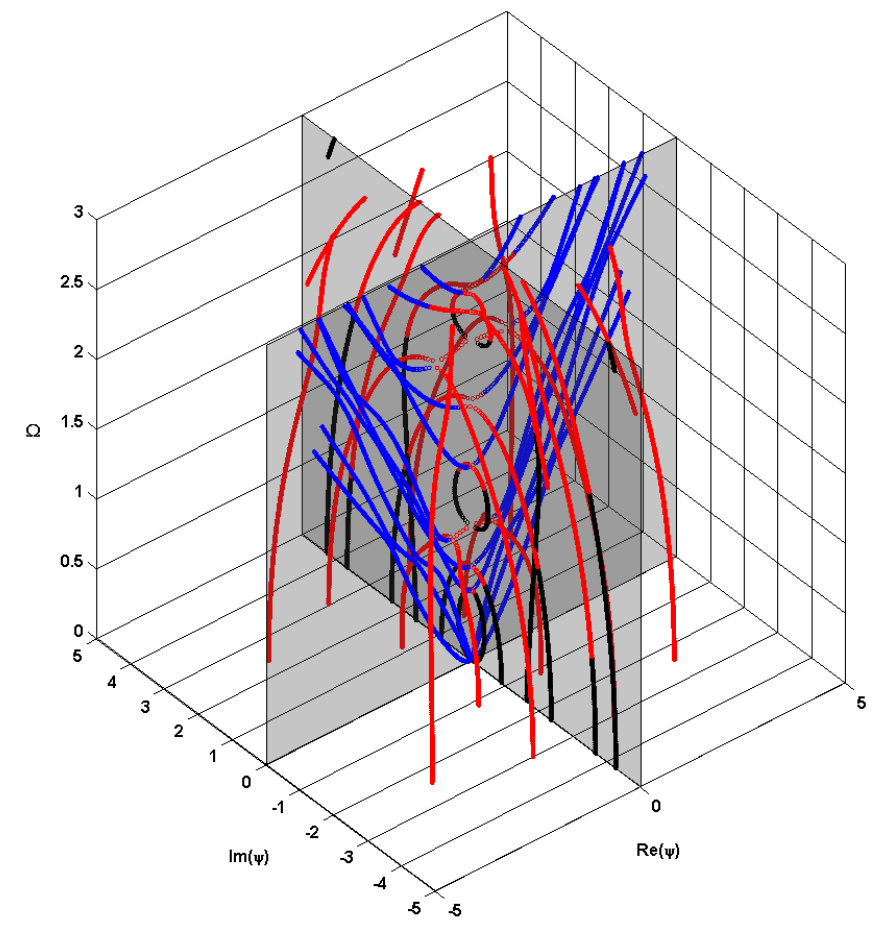

Figure 7: Modes in a free bilayer plate system: $5 \mathrm{~mm}$ thick elastic orthorhombic plate and 3 $\mathrm{mm}$ hysteretic-type viscoelastic triclinic plate computed with the SCM. Propagation along the $\{\mathrm{Z}\}$ axis and the $\{\mathrm{Y}\}$ axis perpendicular to the plane of the plate. Propagating Modes: low attenuated modes in blue, highly attenuated modes in red and almost imaginary wavenumber modes in black. Plot non-dimensional axes: $\Omega=h \omega / \pi V_{66}$ and $\Psi=h k / \pi$. 


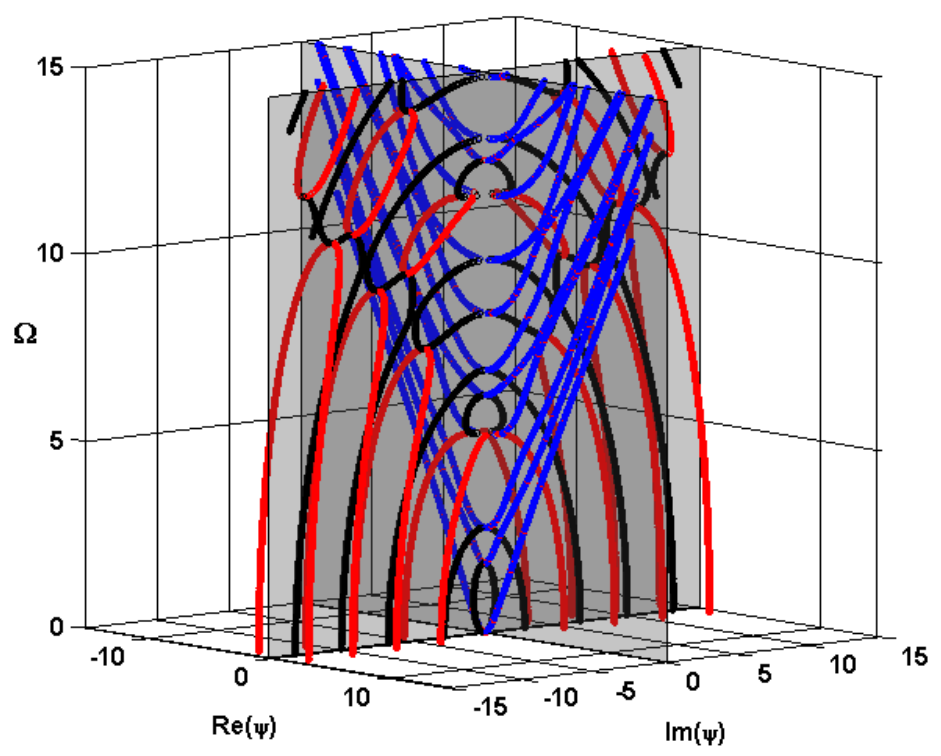

Figure 8: Flexural $\mathrm{n}=1$ modes in free elastic steel rod with radius $r=20 \mathrm{~mm}$. This agrees well with the corresponding 2D plot first published by Pao [28]. Wave propagation along the $\{\mathrm{z}\}$ axis of the cylinder. Propagating modes (lowly attenuated modes) in blue. Non-propagating highly attenuated modes in red and black (purely imaginary). Plot nondimensional axes: $\Omega=a \omega / V_{66}$ and $\Psi=a k$.

The first example shows the dispersion curves for flexural modes with harmonic order $n=1$ in an elastic cylindrical steel rod where the propagation takes place along the axis of the cylinder. Fig. 8 shows the spectrum computed by the SCM which were compared by the authors to the two-dimensional plot in Pao [28] showing excellent agreement. The original figure by Pao has not been included in this paper in order to avoid repetititon of identical figures but the reader can easily find it in the reference provided. As pointed out in the literature [10], it can be seen in Fig. 8 that the incomplete imaginary black branches (non-propagating purely imaginary modes) are linked in three-dimensional space by complex red branches (non-propagating modes with complex wavenumber). Further details about the physical and geometrical properties are in the appendix.

To close this section a final new case is presented. Flexural $n=0$ modes 
(Longitudinal coupled with Torsional) in a hollow cylinder composed of two different layers are studied with propagation along the axis of the cylinder. The innermost layer is a triclinic viscoelastic plate of thickness $h_{o}=3 \mathrm{~mm}$ and the outer layer is an elastic orthorhombic plate of thickness $h_{i}=5 \mathrm{~mm}$ whose fibres along the $\{z\}$ axis have been rotated 45 degrees to form an helicoidal path around the cylinder's axis, the internal radius of the cylinder is $r_{i}=17 \mathrm{~mm}$. The three-dimensional spectrum computed by the SCM is shown in Fig.9 for the first five modes; two-dimensional dispersion curves for the propagating modes have already been shown in the literature for similar systems, see [15], but the full spectrum for anisotropic materials presented here has not been published elsewhere to the best of the authors' knowledge. Due to the presence of an absorbing layer, all modes are propagating: with very low attenuation showing in blue, with highly attenuated modes showing in red and modes with almost imaginary wavenumber showing in black. Note that, strictly speaking, these latter modes should have been coloured in red too. However, since the system contains a perfectly elastic layer some branches show a behaviour closer to that featured in the perfectly elastic case and thus have a very small real part, though not strictly zero. In order to make this more apparent and clear, the authors have chosen to colour them in black for this figure. Again, the triclinic layer has been chosen because of its generality, thus showing that the SCM can provide the complete threedimensional spectrum for the most general cases in cylindrical geometry. It is notable that with advances in technology it is these complicated multilayer and generally anisotropic cases that now require modelling.

\section{Discussion of Results and Conclusions}

The capability of the SCM approach to find full three-dimensional solutions for dispersion curves has been demonstrated here. The SCM has computed the complete spectrum of various known cases, as well as some new settings, efficiently and accurately, without modifying the algorithm, to find all the eigenvalues. This advantage of not missing any modes has been repeatedly emphasized in the literature in other contexts ([16] or [20]) and in previous papers on guided waves ([12], [14] or [15]), the interested reader can find a detailed discussion about this and other advantages of the SCM in those references.

The SCM throws new light onto problems involving elastic or viscoelastic 


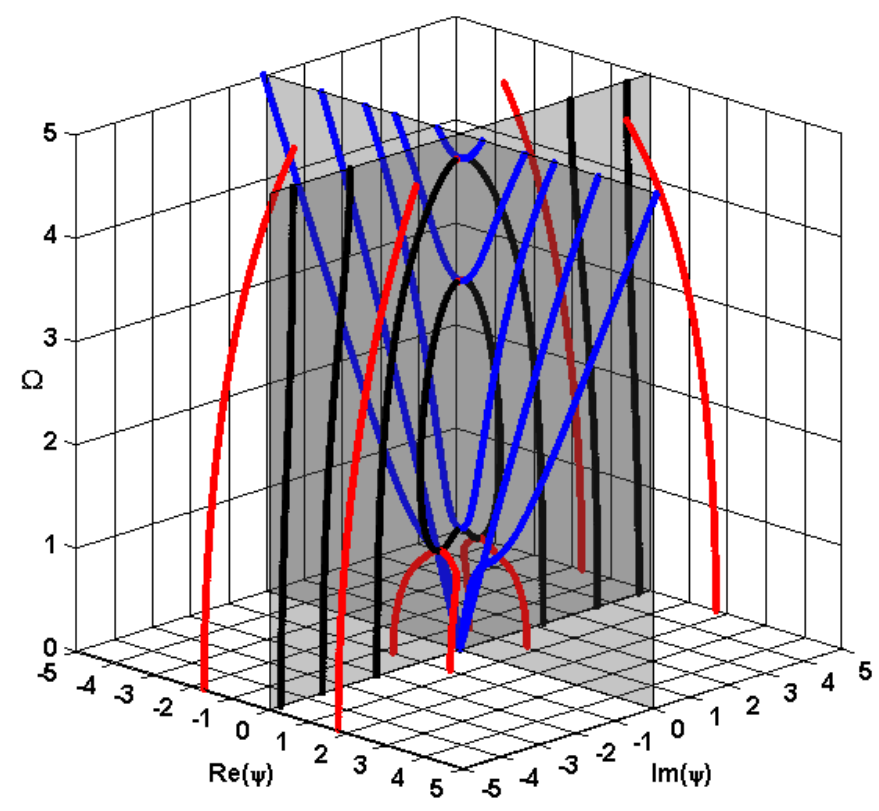

Figure 9: Flexural $\mathrm{n}=0$ modes in free two-layered cylinder. The inner layer is a kelvinvoigt-type viscoelastic triclinic crystal of thickness $h_{i}=5 \mathrm{~mm}$ and the outer layer is an elastic orthorhombic crystal of thickness $h_{o}=3 \mathrm{~mm}$ whose fibres along the $\{\mathrm{z}\}$ axis have been rotated 45 degrees to form an helicoidal path around the cylinder's axis. The cylinder's inner radius is $r_{i}=17 \mathrm{~mm}$. Propagation along the $\{\mathrm{z}\}$ axis of the cylinder. Propagating Modes: low attenuated modes in blue, highly attenuated modes in red and almost imaginary wavenumber modes in black. Plot non-dimensional axes: $\Omega=a \omega / V_{66}$ and $\Psi=a k$.

media which are often very demanding for conventional approaches (rootfinding routines or FE simulations), for instance, for a two-layer system of isotropic and orthorhombic viscoelastic materials. For those cases where the root-finding routines work, one often finds that the solutions are incomplete and the path of the dispersion curves is unclear thus giving the impression of mode merging and similar unusual phenomena. This troublesome situation can sometimes be elucidated by tedious work refining the search algorithm to complete the missing parts in a three-dimensional space and get the full picture of the spectrum. FE simulations are a good and useful alternative to root-finding routines and can also model any material and configuration. However, FE simulations require a far greater coding effort, understanding 
of the methodology and a careful examination of the solutions to discard the spurious unphysical modes; a more detailed discussion about this is in [15]. It is seen that the SCM yields the complete solution straightforwardly; only an adequate post processing of the results is needed to clearly plot the different parts of the spectrum according to the nature of the wavenumber.

In addition to the classical examples from the literature, the SCM has successfully computed the complete three-dimensional spectrum of the most general and complicated cases which, to the best of the authors' knowledge, had not been presented before. Even though similar general cases have been solved recently by Hernando et. al [14] for perfectly elastic media and by Hernando, Fan et. al for viscoelastic materials [15], they focus solely on lowly attenuated modes and their corresponding two-dimensional dispersion curves. Therefore, it is hoped that the investigations presented here contribute to complete the picture in the most general and until now unknown cases.

The general examples involving triclinic materials presented earlier have highlighted the need of a clarification regarding the symmetries of crystals and the consequences on the waves propagating within them. In the literature, the symmetry properties of the Lamb dispersion equations are often used for simplification or to draw certain conclusions about the nature of the solution. For instance in [4] they take advantage of the symmetry $k \leftrightarrow-k$ to reduce the dimension of the matrix. Another example of this assertion can be found in Graff [10] (chapter 8, page 455). From this, one might draw the erroneous conclusion that when the dispersion relation lacks that symmetry, so will its solutions. Triclinic materials' dispersion relation, for instance, lack this symmetry as shown in [25]. In the course of the present investigation one can see that in spite of the absence of this symmetry in triclinic materials, its solution shows this $k \leftrightarrow-k$ symmetry. This apparent paradox is solved by noticing that the mentioned symmetry has a deeper source than the formal structure of the dispersion relation: all crystal classes possess one centre of symmetry, see for example [29], which means that they are invariant under the change $\vec{r} \leftrightarrow-\vec{r}$, which in general is not the same as a mirror symmetry through the plane normal to the direction of the wave vector. Therefore, regardless of the formal structure of the dispersion relation, every crystal will display that intrinsic symmetry as the examples in the present work reveal. 
Apart from the observation made in the previous paragraph, it has also been pointed out and explained at the end of section III why the modes of the same symmetry family in a free viscoelastic plate do not cross. This apparent crossing is merely a visual effect of the projection of the dispersion loci onto a two-dimensional plane which is readily clarified when one looks at the full three-dimensional solution and realizes that for each independent family there is only one dispersion relation, the very same as in the elastic case, which cannot be further factorized. It is also important to notice the following: one might argue that the example presented in Fig.1 is actually a perfect counterexample for the statement given in section III, namely, that having only one dispersion relation which cannot be further factorized for each family, no crossings can occur amongst modes within the same family. Careful consideration shows that this is not the case. As explained by Auld [8] (Chapter 10, Volume II), at these "crossing points" (also termed cutoff points in the literature), rightgoing and leftgoing modes meet. These modes correspond to different branches of the square root of the imaginary wavenumber entering the dispersion relations and are physically distinguishable by energy arguments $([8,6])$ Therefore they must be considered as different families: symmetric rightgoing Lamb modes and symmetric leftgoing Lamb modes for instance. Unfortunately, bringing this to the fore by deriving explicit expressions for both branches of the complex wavenumber in terms of the angular frequency within a given family of modes is, to the best of the authors' knowledge, only possible for SH modes but not for the rest due to the complexity of the dispersion relations. This distinction has been achieved numerically, though no details are given, in [4] for symmetric Lamb modes and their results confirm that for a given branch, within a given family of independent modes, strictly no crossings are seen in the dispersion loci. For the viscoelastic case, as pointed out by Auld [8] not cutoff points exist and the problem of the crossings disappears.

The progress made here is vital as it tackles a fundamental issue, the accurate $3 \mathrm{D}$ representation of complex modes that also arises in other areas, most notable for the equally important leaky mode cases where energy leakage into the surrounding medium contributes to the attenuation of the waves. In those cases this also modifies the dispersion loci demanding that the complete solution must be visualised in three-dimensional space for better understanding, [5]. This, unfortunately, is not a simple modification as it requires substantial modifications to account for the infinite nature of the surround- 
ing embedding layers, but our work here does tackle a key point required for this allied leaky mode problem.

\section{Appendix A. Physical properties and numerical data}

The physical properties of the materials used for the figures are given here. The number of grid points $N$ varies from one example to another, but

it is always at least double the number of modes plotted in the figure to ensure spectral accuracy.

The physical properties for the steel plate whose dispersion curves are shown in Fig.1 are:

$$
\rho=7932 \mathrm{~kg} / \mathrm{m}^{3} ; \quad c_{L}=5960 \mathrm{~m} / \mathrm{s} ; \quad c_{S}=3260 \mathrm{~m} / \mathrm{s}
$$

For Fig.2.a and Fig.3 the viscoelastic steel plate has the following properties:

$$
\rho=7932 \mathrm{~kg} / \mathrm{m}^{3} ; \quad c_{L}=5960 \mathrm{~m} / \mathrm{s} ; \quad c_{S}=3260 \mathrm{~m} / \mathrm{s}
$$

with the viscoelastic matrix given in terms of the isotropic stiffness matrix by:

$$
\eta_{i j}=0.015 c_{i j}
$$

The monoclinic plate of Fig.4, Fig.5 and Fig.6 has the following properties:

$$
\rho=1500 \mathrm{~kg} / \mathrm{m}^{3} ; \quad h=3 \mathrm{~mm}
$$

The elastic stiffness matrix is given in GPa:

$$
c=\left(\begin{array}{cccccc}
21.93 & 5.85 & 26.05 & & 14.33 & \\
& 12.30 & 6.55 & & 0.61 & \\
& & 81.88 & & 37.59 & \\
& & & 5.44 & & 1.22 \\
& & & & 26.36 & \\
& & & & & 4.03
\end{array}\right)
$$


The viscosity matrix in $\mathrm{MPa}$ is:

$$
\eta=\left(\begin{array}{cccccc}
66.44 & 16 & 81.81 & & 39.30 & \\
& 37 & 6 & & -8.66 & \\
& & 244.94 & & 115.29 & \\
& & & 17.25 & & 4.76 \\
& & & & 80.81 & \\
& & & & & 11.75
\end{array}\right)
$$

The multilayer system of Fig.7 is composed of two layers. The top layer has the following properties:

$$
\rho=1500 \mathrm{~kg} / \mathrm{m}^{3} ; \quad h=5 \mathrm{~mm}
$$

The elastic stiffness matrix is given in GPa:

$$
c=\left(\begin{array}{cccccc}
132 & 6.90 & 5.90 & & & \\
& 12.30 & 5.50 & & & \\
& & 12.10 & & & \\
& & & 3.32 & & \\
& & & & 6.21 & \\
& & & & & 6.15
\end{array}\right)
$$

The bottom layer has the following properties:

$$
\rho=8938.4 \mathrm{~kg} / \mathrm{m}^{3} ; \quad h=3 \mathrm{~mm}
$$

The elastic stiffness matrix is given in GPa:

$$
c=\left(\begin{array}{cccccc}
2.08 & 1.09 & 0.93 & 0.16 & -0.16 & -0.23 \\
& 1.68 & 1.36 & -0.25 & 0.11 & 0.09 \\
& & 1.86 & 0.08 & 0.08 & 0.14 \\
& & & 1.00 & 0.14 & 0.06 \\
& & & & 0.35 & 0.16 \\
& & & & & 0.59
\end{array}\right)
$$

The viscosity matrix in GPa is given by:

$$
\eta_{i j}=0.025 c_{i j}
$$


The parameters for the steel cylindrical rod of Fig.8 are as follows:

$$
\rho=7932 \mathrm{~kg} / \mathrm{m}^{3} ; \quad r=20 \mathrm{~mm} ; \quad c_{L}=5960 \mathrm{~m} / \mathrm{s} ; \quad c_{S}=3260 \mathrm{~m} / \mathrm{s}
$$

$r$ stands for the radius of the rod.

In Fig.9 dispersion curves for a cylinder composed of two different layers is presented. The outer layer is an hexagonal (transversely isotropic) elastic material which has been rotated so that the fibres follow an helical path around the cylinder, it has the following properties:

$$
\rho=1605 \mathrm{~kg} / \mathrm{m}^{3} ; \quad h=3 \mathrm{~mm} ; \quad \phi_{r}=\frac{\pi}{4}
$$

where $\phi_{r}$ is the angle of rotation with respect to the $\{r\}$ axis. The elastic stiffness matrix is given in GPa:

$$
c=\left(\begin{array}{cccccc}
11.69 & 5.85 & 5.62 & & & \\
& 11.69 & 5.67 & & & \\
& & 130.19 & & & \\
& & & 3.70 & & \\
& & & & 3.70 & \\
& & & & & \frac{c_{11}-c_{12}}{2}
\end{array}\right)
$$

The inner layer is a viscoelastic triclinic material whose properties are:

$$
\rho=1500 \mathrm{~kg} / \mathrm{m}^{3} ; \quad h=5 \mathrm{~mm} ; \quad r_{i}=17 \mathrm{~mm}
$$

$h$ stands for the thickness of the plate and $r_{i}$ is the inner radius of the cylinder. The elastic stiffness matrix is given in GPa:

$$
c=\left(\begin{array}{cccccc}
74.29 & 28.94 & 5.86 & 0.20 & -0.11 & 37.19 \\
& 25.69 & 5.65 & 0.09 & -0.08 & 17.52 \\
& & 12.11 & 0.01 & -0.01 & 0.22 \\
& & & 4.18 & 1.31 & 0.09 \\
& & & & 5.35 & -0.07 \\
& & & & & 28.29
\end{array}\right)
$$


The viscosity matrix in $\mathrm{MPa}$ is:

$$
\eta=\left(\begin{array}{cccccc}
218 & 76.50 & 16.40 & -3.60 & 0.69 & 116 \\
& 71.10 & 19.20 & -0.77 & 2.15 & 50 \\
& & 42.20 & -0.96 & 0.63 & -3.07 \\
& & & 11.10 & 2.89 & -1.15 \\
& & & & 13.60 & 1.48 \\
& & & & & 93.50
\end{array}\right)
$$

The above material's stiffness matrix has been generated by two successive rotations of an orthorhombic material about different axes. An analogous procedure was used by Nayfeh and Chimenti [30] and Li and Thompson [24]. Due to its higher degree of anisotropy and complexity triclinic materials often feature negative entries, see [31] for another example of triclinic elastic constants for albite.

\section{Appendix B. References}

[1] R. D. Mindlin, Waves and vibrations in isotropic, elastic plates, Structural Mechanics. (Eds. J.N. Goodier and N. Hoff) (1960) 199-323.

[2] M. Onoe, H. D. McNiven, R. D. Mindlin, Dispersion of axially symmetric waves in elastic rods, Journal of Applied Mechanics 29 (4) (1962) 729734.

[3] R. Kumar, Dispersion of axially symmetric waves in empty and fluidfilled cylindrical shells, Geophysics 27 (1972) 317-329.

[4] V. Pagneux, A. Maurel, Determination of Lamb mode eigenvalues, Journal of the Acoustical Society of America 110(3) (2001) 1307-1314.

[5] S. Rokhlin, D. Chimenti, A. Nayfeh, On the topology of the complex wave spectrum in a fluid-coupled elastic layer, Journal of the Acoustical Society of America 85 (3) (1989) 1074-1080.

[6] F. Simonetti, M. Lowe, On the meaning of Lamb mode nonpropagating branches, Journal of the Acoustical Society of America 118(1) (2005) 186-192. 
[7] I. Bartoli, A. Marzani, F. Lanza di Scalea, E. Viola, Modeling wave propagation in damped waveguides of arbitrary cross-section, Journal of Sound and Vibration 295 (2006) 685-707.

[8] B. A. Auld, Acoustic Fields and Waves in Solids, 2nd Ed., Krieger Publishing Company, Florida, 1990, pp. 1-878.

[9] M. Castaings, E. Le Clezio, B. Hosten, Modal decomposition method for modeling the interaction of lamb waves with cracks, Journal of the Acoustical Society of America 112 (6) (2002) 2567-2582.

[10] K. F. Graff, Rayleigh and Lamb Waves, Dover, New York, 1991, pp. $1-649$.

[11] J. L. Rose, Ultrasonic Waves in Solid Media, Cambridge University Press, Cambridge, 1999, pp. 1-476.

[12] A. Adamou, R. Craster, Spectral methods for modelling guided waves in elastic media, Journal of the Acoustical Society of America 116 (3) (2004) 1524-1535.

[13] S. Towfighi, T. Kundu, M. Ehsani, Elastic wave propagation in circumferential direction in anisotropic cylindrical curved plates, Journal of Applied Mechanics 69 (2002) 283-291.

[14] F. Hernando Quintanilla, M. Lowe, R. Craster, Modelling guided elastic waves in generally anisotropic media using a spectral collocation method, Journal of the Acoustical Society of America 137 (3) (2015) 1180-1194.

[15] F. Hernando Quintanilla, Z. Fan, M. Lowe, R. Craster, Guided waves' dispersion curves in anisotropic viscoelastic single- and multi-layered media, Proceedings of the Royal Society A In press.

[16] L. Trefethen, Spectral Methods in MATLAB, SIAM, Philadelphia, 2000, pp. 1-181.

[17] J. Weideman, S. Reddy, A matlab differentiation matrix suite, ACM Transactions on Mathematical Software 26 (4) (2000) 465-519.

[18] T. Bridges, P. Morris, Differential eigenvalue problems in which the parameter appears nonlinearly, Journal of Computational Physics 55 (1984) 437-460. 
[19] J. A. Postnova, Trapped modes in non-uniform elastic waveguides: asymptotic and numerical methods, Ph.D. thesis, Imperial College London (2008).

[20] J. P. Boyd, Chebyshev and Fourier Spectral Methods, Dover, New York, 2001, pp. 1-688.

[21] R. Fiorito, W. Madigosky, H. Überall, Resonance theory of acoustic waves interacting with an elastic plate., Journal of the Acoustical Society of America 66 (6) (1979) 1857-1866.

[22] H. Überall, B. Hosten, M. Deschamps, A. Gerard, Repulsion of phasevelocity dispersion curves and the nature of plate vibrations., Journal of the Acoustical Society of America 96 (2) (1994) 908-917.

[23] M. F. Werby, H. Überall, The analysis and interpretation of some special properties of higher order symmetric lamb waves: The case for plates.., Journal of the Acoustical Society of America 111 (6) (2002) 2686-2691.

[24] Y. Li, R. B. Thompson, Influence of anisotropy on the dispersion characteristics of guided ultrasonic plate modes, Journal of the Acoustical Society of America 87 (5) (1990) 1911-1931.

[25] A. H. Nayfeh, D. E. Chimenti, Free wave propagation in plates of general anisotropic media, Journal of the Applied Mechanics 56 (1989) 881-886.

[26] L. P. Solie, B. A. Auld, Elastic waves in free anisotropic plates, Journal of the Acoustical Society of America 54 (1) (1973) 50-65.

[27] S. Rokhlin, D. Chimenti, P. Nagy, Physical Ultrasonics of Composites, Oxford University Press, Oxford, 2011, pp. 1-378.

[28] Y.-H. Pao, The dispersion of flexural waves in an elastic circular cylinder, part ii, Journal of Applied Mechanics 29 (1962) 61-64.

[29] E. Dieulesaint, D. Royer, Elastic Waves in Solids, Springer Verlag, Berlin Heidelberg, 1996, pp. 1-392.

[30] A. H. Nayfeh, D. E. Chimenti, Free wave propagation in plates of general anisotropic media, in: D. Thompson, D. Chimenti (Eds.), Review of Progress in Quantitative Non-destructive Evaluation, Plenum Press, New York, 1989, p. 181. 
[31] J. M. Brown, E. H. Abramson, R. J. Angel, Triclinic elastic constants for low albite, Physics and Chemistry of Minerals 33 (4) (2006) 256-265. 